\title{
When More Is Less and Less Is More: The Role of Ideal Point Availability and Assortment in Consumer Choice
}

\author{
ALEXANDER CHERNEV*
}

\begin{abstract}
Contrary to the common wisdom that more choice is always better, selections made from large assortments can lead to weaker preferences. Building on the extant literature, this research identifies ideal point availability as a key factor moderating the impact of assortment on choice. It is proposed that, in the case of large assortments, ideal point availability can simplify choice, leading to a stronger preference for the selected alternative. In contrast, for choices made from smaller assortments, ideal point availability is proposed to have the opposite effect, leading to weaker preferences. Data obtained from four experiments lend support for the theory and the empirical predictions advanced in this article.
\end{abstract}

\begin{abstract}
A common assumption in marketing is that assortment benefits consumers. This assumption is consistent with the prediction by classic economic theories that larger assortments should always be beneficial for consumers because they provide for a potentially better match between consumers' own preferences and the product offering. Recent research, however, has suggested that increasing the size of the choice set may have adverse consequences on the strength of preferences because it can confuse consumers, increasing the probability of delaying their choice or not choosing at all (Dhar 1997; Greenleaf and Lehmann 1995; Iyengar and Lepper 2000).

Most of the recent decision research has focused on demonstrating the adverse impact of large assortments on choice without explicitly identifying conditions moderating this effect. In this context, the goal of this article is to identify factors that determine when large product assortments will strengthen consumer preferences, as predicted by traditional economics literature, and when large assortments will weaken preferences, as suggested by recent findings in the behavioral decision literature.

Building on the constructive view of preferences (Bettman, Luce, and Payne 1998; Simonson and Tversky 1992), this research argues that choices from different size assortments are a function of the degree to which consumers have

*Alexander Chernev (ach@nwu.edu, phone: 847-467-4095, fax: 847491-2498) is associate professor of marketing, Kellogg School of Management, Northwestern University, 2001 Sheridan Road, Evanston, IL 60208. The author thanks Bobby Calder, Gregory Carpenter, Michaela Draganska, Dawn Iacobucci, Brian Sternthal, Alice Tybout, the editor, associate editor, and the three anonymous reviewers for their valuable comments and assistance. This research has also benefited from seminars at Dartmouth University, UCLA, and the University of Chicago.
\end{abstract}

a readily available ideal attribute combination. In particular, it is proposed that, when choosing from a larger assortment, consumers with an available ideal point are likely to have stronger preferences for the chosen option than consumers without an available ideal attribute combination. In contrast, when choosing from a smaller assortment, consumers with an available ideal point are likely to have weaker preferences for the chosen option than consumers without an available ideal attribute combination. The psychological mechanism of how the availability of an ideal attribute combination moderates the impact of assortment on consumer preferences is discussed in more detail in the next sections.

\section{THEORETICAL BACKGROUND}

Extant research has identified several factors that could potentially increase the strength of consumer preferences in the context of larger assortments. The most intuitive factor, featured prominently in the economics research, is that larger assortments offer an opportunity for a better match between an individual's preferences and the characteristics of the alternatives in the choice set (see Lancaster 1990 for a review).

It has also been proposed that larger assortments also might lead to stronger preferences because they offer option value (Reibstein, Youngblood, and Fromkin 1975) and allow consumers to maintain flexibility when making a purchase decision (Kahn and Lehmann 1991; Kreps 1979; March 1978; see also Ratner, Kahn, and Kahneman 1999). In this context it has been suggested that consumers often prefer larger assortments in anticipation of future variety-seeking behavior (McAlister 1982; Pessemier 1978; Simonson 1990; Walsh 1995). It has been further suggested that in addition 
to the variety effect, consumers might experience additional utility simply from having multiple items in the choice set (Kahn, Moore, and Glazer 1987; see also Broniarczyk, Hoyer, and McAlister 1998), a proposition consistent with the view that larger assortments might influence preferences by creating a perception of freedom of choice (Brehm 1972).

Finally, it has been argued that larger assortments affect consumer preferences by reducing the uncertainty of whether the choice set at hand adequately represents all potentially available options. Recent experiments show that consumers may delay their purchasing because they are unaware of the distribution of potential alternatives and are uncertain of the degree to which the available set is representative of the entire set of possible options (Greenleaf and Lehmann 1995; Karni and Schwartz 1977). To illustrate, consumers might feel more confident when selecting from a retailer that offers a larger assortment because it is less likely that a potentially superior alternative is not represented in the available choice set.

All these factors might contribute to the overall positive impact of product assortment on choice and strengthen consumer preferences. Yet, one can argue that large assortments might also lead to weaker preferences because of increased demand on an individual's cognitive resources associated with the extra effort required to evaluate the attractiveness of alternatives in the large assortment (Huffman and Kahn 1998; Jacoby, Speller, and Kohn 1974; Scammon 1977; Shugan 1980). It has further been argued that increasing the size of the choice set might confuse consumers, leading to weaker preferences and lower choice probability (Dhar 1997; Greenleaf and Lehmann 1995; Iyengar and Lepper 2000; Malhotra 1982; see Simonson 1999 for a review).

Most of the research discussing the adverse impact of assortment on choice focuses on the extra effort needed to evaluate the alternatives in a large assortment. In doing so, it is implicitly assumed that consumers have readily available criteria for evaluating choice alternatives and that their main task is to find the alternative that best matches these criteria. Yet, consumers often make choices in areas where they lack sufficient expertise and consequently do not have readily available decision criteria. In such cases consumers must first construct their attribute preferences in order to evaluate the alternatives in the set - a task that can be more easily accomplished in the context of a smaller rather than a larger assortment. The impact of the availability of readily articulated attribute preferences on how product assortment affects choice is discussed in more detail in the next section.

\section{PRODUCT ASSORTMENT AND IDEAL POINT AVAILABILITY}

Consumers often approach decision problems with already formed preferences for a particular alternative and, provided that this alternative is available, are likely to make a selection without detailed evaluation of the other options. On many occasions, however, consumers do not have a preferred option, or their preferred option is not available.
To illustrate, consider three consumers who are choosing from the same set of alternatives, in this case chocolates.

Consumer A is familiar with the different chocolate products and already has a favorite product, Godiva Medallion (vanilla-flavored solid white chocolate). Consumer B is not familiar with the Godiva brand, yet she is aware of the key attributes describing chocolates and has an ideal combination of these attributes. Say, she prefers white chocolate to dark or milk chocolate, solid chocolate to chocolates with various fillings, vanilla flavor to other flavors, and does not like chocolate with nuts. Additionally, cocoa content is the most important attribute for her, followed by chocolate type, then flavor and nut content. Finally, consumer $\mathrm{C}$ is aware of the key attributes describing chocolates but has neither a favorite brand nor a readily available ideal attribute combination.

Consumers A, B, and $\mathrm{C}$ in the above example differ in terms of the availability of an ideal point, defined as a combination of attributes and attribute values describing the ideal choice alternative (Carpenter and Nakamoto 1989). In this context, consumer A has a readily defined ideal point and a specific option (Godiva Medallion) corresponding to that point. Furthermore, because consumer B has articulated within-attribute preferences as well as articulated importance weights associated with these attributes, she also has a readily available ideal point even though she has not identified a specific alternative as the optimal choice. It is important to note that the decision process of consumer $\mathrm{A}$ is likely to differ substantially from the decisions made by consumers $\mathrm{B}$ and $\mathrm{C}$, because consumer $\mathrm{A}$ has a memorized preference for one of the choice options, whereas consumers $\mathrm{B}$ and $\mathrm{C}$ are yet to construct option-specific preferences. Thus, consumer A's strategy, also known as affect referral (Wright 1975), involves simply retrieving a memorized evaluation of the alternatives without actively processing the attributes and attribute levels of the choice options.

This research focuses on scenarios in which consumers are faced with decision problems for which affect referral does not provide a ready solution. Specifically, it examines how product assortment affects decision behavior when consumers are faced with a choice problem of some novelty and complexity. In the above example, the focus of this research is on the differential impact of product assortment on the strength of preferences for consumers B and C.

A key proposition of this research is that the impact of assortment on choice is a function of the degree to which consumers have an available ideal point. This proposition is based on the notion that decision processes are contingent on the degree of articulation of the readily available preferences. To make a choice, consumers with a readily available ideal point must evaluate available alternatives in search of the option with the highest utility derived from these preferences. In contrast, consumers without a readily available ideal point must first articulate their attribute preferences in order to identify the optimal alternative. As a result, consumers without an ideal point face the structurally more difficult task of simultaneously forming their ideal attribute 
combination and searching for the option that best matches their ideal point.

It is predicted, therefore, that in larger assortments the availability of an articulated ideal point is likely to be associated with stronger preferences for the chosen alternative. Indeed, prior research has shown that the cognitive costs of evaluating choice alternatives depend upon the number of alternatives to be considered (Payne 1976; Shugan 1980). In cases where choice alternatives are unique (i.e., no replicates), increasing the set size by default leads to an increase in the number of relevant attributes to be considered. Because there are more attributes to be evaluated and potentially incorporated in the decision criteria, the choice process is likely to be more complicated in the context of larger assortments. As a result, when faced with a larger assortment, consumers without a readily available ideal point are likely to face a rather complex two-stage decision, which might, in turn, lead to weaker preferences for the selected alternative. In contrast, consumers with a readily available ideal point can focus their effort on the relatively simpler process of searching for the option that best matches their ideal attribute combination.

It is further predicted that in smaller assortments the availability of an articulated ideal point can have the opposite result, leading to weaker preferences for the chosen alternative. Indeed, if consumers with articulated preferences are simply trying to map their ideal attribute combination onto the available choice alternatives, then, in cases where the assortment is small, the chances of a match are rather low, which is likely to lead to a choice perceived to be suboptimal. In contrast, consumers without an available ideal attribute combination construct their preferences based on the available assortment and, as a result, their preferences are more likely to reflect the available alternatives in the set. Therefore, it is argued that the availability of an ideal point does not always strengthen preferences; for choices from small assortments, readily available ideal points can actually have the opposite effect.

To summarize, it is argued that the availability of an ideal attribute combination moderates the impact of assortment on consumer strength of preferences. Consumers without a readily available ideal point are faced with the dual task of articulating their own ideal attribute combination and identifying the alternative that best matches their available ideal point. The difficulty of this dual task is further complicated for larger assortments because of the increased number of attributes, attribute levels, and options to be considered. In this context, choices from larger assortments are likely to be associated with weaker preferences for the selected alternative. In contrast, consumers with a readily available ideal point face the simpler task of searching for the best alternative, using their ideal point as a benchmark for evaluating choice options. Increasing the assortment in this case might also complicate their choice, but to a lesser degree than for consumers without an available ideal attribute combination. It is also predicted that for choices from small assortments, the impact of the availability of an articulated ideal attribute combination is reversed-consumers with an articulated ideal point are likely to have weaker preferences for the chosen alternative relative to consumers without an articulated ideal point.

More formally, these predictions can be expressed as follows:

H1: The availability of an articulated ideal point moderates the impact of assortment on consumer preferences.

H2: When choosing from a larger assortment, consumers with an available ideal point are likely to have stronger preferences for the chosen option than consumers without an available ideal attribute combination.

H3: When choosing from a smaller assortment, consumers with an available ideal point are likely have to weaker preferences for the chosen option than consumers without an available ideal attribute combination.

These predictions are empirically tested in a series of four experiments. Experiment 1 examines the core proposition that ideal point availability moderates the impact of assortment on consumer preferences. Experiments 2 and 3 explicitly investigate the role of ideal point availability by focusing on how the availability of articulated attribute trade-offs moderates the impact of assortment on choice. Finally, experiment 4 offers further support for the experimental predictions by using alternative manipulation procedures and by offering converging process measures.

\section{EXPERIMENT 1}

The goal of this experiment was to examine the impact of ideal point availability on the strength of consumer preferences as a function of assortment size. Respondents were first asked to articulate their ideal attribute combination and were then asked to make a choice from either a larger or a smaller assortment. The dependent variable, strength of preferences, was operationalized as the likelihood of switching to another option. The method, results, and a discussion of the findings of this experiment are presented in more detail next.

\section{Method}

Ideal Point Availability. Prior to being presented with the actual choice set, respondents were asked to indicate the most attractive level of each attribute and to rank order the attributes in terms of their relative importance. This manipulation is similar to the compositional preference-elicitation methods often used to reveal systematic components that underlie consumer product evaluations. Specifically, compositional elicitation methods ask respondents to rank order interval-scaled levels within each attribute and rate the importance of the difference between the best and the worst 
levels of an attribute. The importance ratings are then multiplied by the preference orders and used as a starting point for subsequent paired judgments (Green et al. 1991; Huber et al. 1993). The ideal point articulation approach used in this research is similar to preference-elicitation procedures in that it asks consumers to form judgments about their attribute preference and attribute importance. The key difference is in the ultimate goal of the elicitation task. Traditionally, preference elicitation is used to measure consumer preferences; as a result, it requires consumers to provide a detailed account of their preferences. In contrast, the elicitation procedure in this experiment aims to help consumers articulate their own preferences. Therefore, respondents were asked to select only the most attractive level for each of the attributes and simply rank order these attributes without explicitly generating ratings for all attributes and all attribute levels.

This difference between the traditional self-explicated conjoint measurement and the manipulation used in this experiment is important for two reasons. First, whereas the self-explicated format has been shown to focus inordinate attention on less important attributes (Green and Srinivasan 1990; Tversky and Kahneman 1974), the manipulation used in this research is more similar to the noncompensatory format of many of the decision heuristics used in choice. Furthermore, decision tasks in which consumers are explicitly asked to indicate how much of one attribute they are willing to give up in order to gain a better performance on another attribute have been shown to require significant cognitive effort (Huffman and Kahn 1998) and reduce satisfaction with the decision (Garbarino and Edell 1997; Kottemann and Davis 1991). The preference-elicitation task used in this experiment was designed to approximate a choice task while avoiding the potential negative impact of a complex learning task prior to choice.

Product Assortment. Product assortment was manipulated by varying the number of alternatives in the choice set. There were two types of sets: a small assortment set with four alternatives, and a large assortment set with 16 alternatives. The choice of four and 16 alternatives to represent the small and large assortments is consistent with the research on cognitive overload, which demonstrates that individuals can optimally process a maximum of up to six alternatives (Bettman 1979; Malhotra 1982; Wright 1975). This manipulation is also consistent with the findings in cognitive psychology that the processing capacity of the short-term memory is approximately seven chunks of information (see Miller 1956 for a review).

All options in the small assortment sets were also available in the large assortment sets; there were no identical options (replicates) in either of the sets. The options in the small set were described on four attributes-two attributes on which options had different values and two attributes with values that were identical for all alternatives. Options in the large set were also described on four attributes, but there was more variance across attributes. To account for the possibility of confounding the choice set size with the attribute structure of the alternatives (Hoch, Bradlow, and Wansink 1999), two variants of the larger assortment were used. Alternatives in one of the sets varied on two of the four attribute dimensions, with each of these two attributes having four levels. Alternatives in the other set varied on four attributes, with each of the four attributes having two levels. These two sets were counterbalanced across respondents.

Stimuli and Experimental Procedure. The stimuli consisted of various chocolate assortments. Similar stimuli have been successfully used in prior research to examine the impact of variety on consumer preferences (Iyengar and Lepper 2000). Stimuli were described on four attributes, each with four levels: chocolate type (solid chocolate, truffle, praline, caramel); cocoa content (dark chocolate, milk chocolate, white chocolate, espresso chocolate); flavor (original, vanilla, strawberry, cherry); and nut content (no nuts, almonds, hazelnuts, walnuts).

Eighty-eight Northwestern University students were presented with the conditions of a 2 (ideal point availability) $\times 2$ (assortment) between-subjects experimental design. Some of the respondents were initially presented with the preference-articulation procedure and were instructed to think about their ideal chocolate. They were then asked to indicate the most attractive level of each of the attributes describing the choice alternatives and to rank order these attributes. The remaining respondents were directly presented with the choice set without being asked to articulate their preferences.

Next, respondents were asked to consider a particular chocolate assortment and indicate the most attractive alternative. Upon making their selection, respondents were given an option to reaffirm their selection by choosing a chocolate as a reward for their participation in the experiment. Finally, respondents were asked to indicate their perceptions of the variety in the choice set and were also asked several questions regarding their chocolate consumption habits.

Dependent Variable. The key dependent variable, strength of preferences, was operationalized in terms of consumer propensity to switch. As a reward for participating in the experiment, respondents were asked to choose between a small box containing two chocolates of the exact same kind that they selected in the preceding choice task and a small box containing two of the most popular chocolates from the entire Godiva collection. The rationale for this measure is that respondents who had stronger preferences and were confident in their choice were more likely to select the initially chosen alternative when given the opportunity to substitute their selection, while respondents with weaker preferences would be more likely to switch to the most popular option.

\section{Results}

Manipulation Check. The effectiveness of the product assortment manipulation is measured by comparing respon- 
dents' perceptions of variety in the choice alternatives across the experimental conditions. Respondents' variety perceptions indicate significant differences between the small and the large assortment sets. The mean evaluation for the small set was $M=3.95$, significantly different from $M=2.34$, the mean evaluation of the variety in the large set (scale: 1 = overwhelming, $2=$ rather extensive, $3=$ adequate, 4 $=$ somewhat narrow, $5=$ very limited; $F(1,87)=84.75$, $p<.001)$. These data show that consumers did perceive the larger set to offer more variety, and that the variety offered by the experimental sets was not perceived to be extreme in either direction.

Switching Behavior. The main dependent variable, strength of preference, was measured through respondents' switching behavior. Respondents who indicated that they would like to receive a box of chocolates of the same kind they had just chosen were considered to have a low propensity to switch, whereas respondents who indicated that they would like to receive a box of chocolates containing the most popular Godiva selection were considered to have a high propensity to switch. Overall, there were 88 observations: 45 in the preference-articulation condition and 43 in the no-articulation condition. A summary of the strength of preferences data as a function of assortment and ideal point availability is presented in figure 1 .

The data show that respondents who were not asked to articulate their ideal attribute combination were less likely to switch when choosing from a smaller set $(9 \%$ of responses) than when choosing from a larger set (38\% of responses). In contrast, respondents who articulated their ideal point were more likely to switch when asked to choose from a smaller rather than larger set. Thus, $27 \%$ of choices from the smaller set resulted in subsequent switching, compared to only $13 \%$ of choices from the large set.

For the purposes of statistical analysis, the switching pattern was examined as a function of ideal point availability, assortment, and their interaction. Log-linear analysis of the data indicates a significant interaction between ideal point availability and the set size $\left(\chi^{2}(1)=6.46, p=.01\right)$. The increase in switching for respondents without an articulated ideal point was significant $\left(\chi^{2}(1)=5.61, p<.05\right)$, indicating a sizable decrease in the strength of preferences for choices from larger sets. For respondents with an articulated ideal point, the difference in the likelihood of switching from the large compared to the small set was directionally opposite to the one observed for respondents who did not articulate their ideal point, and nonsignificant $\left(\chi^{2}(1)=\right.$ 1.45 , NS).

More important, the choice share of the default alternative for choices from the large assortment was significantly greater for respondents without an articulated ideal point compared to those who articulated their ideal attribute combination $\left(38 \%\right.$ vs. $\left.13 \% ; \chi^{2}(1)=3.88, p<.05\right)$. This finding is consistent with hypothesis 2 . In contrast, for choices from a small assortment, the share of the default alternative was lower for respondents without articulated preferences, although marginally nonsignificant $\left(9 \%\right.$ vs. $27 \% ; \chi^{2}(1)=$
FIGURE 1

\section{SWITCHING BEHAVIOR AS A FUNCTION OF ASSORTMENT} AND IDEAL POINT AVAILABILITY (EXPERIMENT 1)

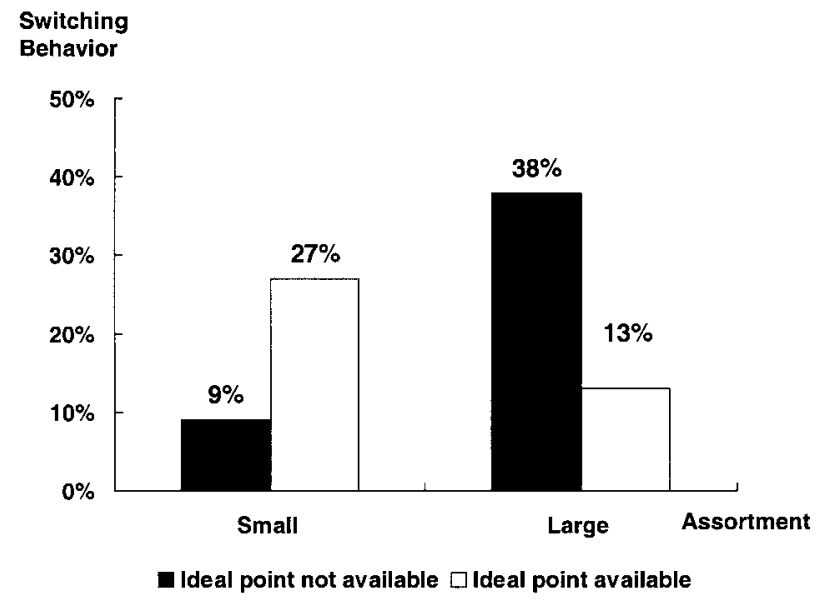

2.59, $p=.11)$. This finding provides directional support for hypothesis 3 .

\section{Discussion}

The data reported in this experiment show that the impact of assortment on consumer preferences is moderated by the availability of an ideal attribute combination (hypothesis 1). For respondents without an articulated ideal point, larger assortments were associated with higher switching probability, whereas for respondents with an articulated ideal point, the effect was in the opposite direction and nonsignificant (hypothesis 2). For choices from a small assortment, the effect of ideal point availability was in the opposite direction, although nonsignificant (hypothesis 3 ).

Experiment 1 examines the impact of product assortment in a context where choice alternatives are described on several distinct attributes. Although such feature-based presentation of choice options is common for many consumer decisions, it can be argued that it implicitly promotes analytical attribute-based processing and an in-depth evaluation of choice alternatives (Tversky 1977). To extend the findings to a scenario where alternatives are processed in a holistic rather than a feature-based manner (Calder 1978; Holbrook and Moore 1981), the findings of experiment 1 were replicated using pictorial representations of the choice alternatives. The design of this replication was identical to the design of the first experiment except that instead of verbally described attributes, pictures of actual chocolates were used as stimuli. The ideal point articulation procedure used pictorial format as well; respondents were instructed to select their most preferred shape of chocolate from the four available shapes (sphere, oval, half-cone, and square), select their most preferred chocolate surface from the four available patterns (smooth, stripe, mesh, and spotted), and select their most preferred chocolate color from the three 
available colors (dark brown, light brown, and white). The data replicated the results reported earlier, thus extending the experimental findings to a broader set of decision scenarios. An additional benefit of this replication was the ability to gain power in data analysis by combining the results from the two experiments (Edgington 1972; Rosenthal 1978). The combined data show that the moderating effect of ideal point availability was significant at the .005 level (hypothesis 1), the difference between respondents with and without articulated preferences for choices made from a larger set was significant at the .01 level (hypothesis 2), and for choices from smaller assortments this difference was significant at the .05 level (hypothesis 3 ). ${ }^{1}$ These data lend further support for the experimental hypotheses.

The theory advanced in this article can be further tested by explicitly examining the role of ideal points in preference articulation. To illustrate, recall consumer B from the prior example. She is aware of the key attributes describing chocolates and has an ideal combination of these attributes: she prefers white chocolate to dark or milk chocolate (the most important attribute), solid chocolate to chocolates with various fillings, vanilla flavor to other flavors, and does not like chocolate with nuts (the least important attribute). Now consider consumer D, who is also aware of the different attributes describing chocolates and has the following preference structure: her favorites are dark and milk chocolate and she dislikes white chocolate, she also likes vanilla-flavored and cherry flavored-chocolate, somewhat likes the original chocolate flavor, and would rather avoid strawberryflavored chocolate.

The key difference between consumers B and D is the dispersion of their attribute-level preferences: both have articulated attribute preferences; however, to make a choice, consumer $\mathrm{D}$ has to trade off the different levels of each attribute, whereas consumer B has already articulated attribute trade-offs. Note also that in this case consumer D has a more comprehensive preference structure: she has articulated her preferences for each level and each attribute describing the choice alternatives, whereas consumer B has a more selective preference structure in which only the top levels of each attribute are articulated.

This example raises the question of whether these different preference structures have the same moderating effect on how assortment affects choice. The ideal point availability argument advanced in this research is based on the notion that ideal points imply a hierarchical attribute structure and readily articulated attribute trade-offs (Carpenter and Nakamoto 1989; Dhar 1997; Simonson and Tversky 1992; Wansink, Kent, and Hoch 1998; see also Bettman and Zins 1979; Biehal and Chakravarti 1982). Because the articulation of attribute trade-offs is also essential for choice (Janis and Mann 1977; Payne, Bettman, and Johnson 1993), the availability of an ideal attribute combination effectively increases the compatibility of consumer preference structures with the decision task, thus reducing the structural

${ }^{1}$ Details on the stimuli and results of this replication are available from the author. complexity of the decision. Therefore, it can be argued that preferences with an articulated ideal point will be more effective in reducing the structural complexity of the decision than will preferences that do not imply an ideal point. Because large assortments are generally associated with more complex decisions, the differential impact of ideal point articulation is likely to be more pronounced for larger than smaller assortments.

The above discussion suggests that the role of ideal point availability can be further tested by comparing two preference-articulation scenarios: one in which consumers comprehensively articulate the attractiveness of each attribute without explicitly articulating an ideal point (e.g., by rating different attributes independently of each other) and one in which consumers articulate their attribute preferences to form an ideal point (e.g., by rating different attributes relative to each other). If the ideal point availability indeed moderates the impact of assortment on choice, then the effects reported in experiment 1 will be less pronounced for consumers who did not explicitly articulate their attribute trade-offs and hence are less likely to have a readily available ideal point. This proposition is experimentally tested in the next experiment.

\section{EXPERIMENT 2}

Experiment 2 was designed to parcel out the effects directly associated with ideal point availability from the more general preference-articulation effects. To accomplish this, two preference-articulation conditions were introduced: a no trade-off evaluation condition and an ideal point condition. Respondents in both conditions were exposed to identical attribute information; the only difference was in the nature of the preference-articulation task.

\section{Method}

Seventy-five Northwestern University students were presented with a 2 (ideal point availability) $\times 2$ (assortment) factorial design. There were 41 respondents in the no tradeoff articulation condition and 34 respondents in the ideal point condition. The experimental procedure was similar to the one used in the first experiment. Respondents were first asked to articulate their preferences, then to make a choice from either a large or a small set, and finally were given an option to switch. In this experiment, all respondents were asked to articulate their preferences, and the articulation procedure varied across subjects.

Respondents in the ideal point condition were presented with an articulation task that required explicit trade-offs. Respondents were first instructed to think about their ideal chocolate, then to indicate the most attractive level of each of the attributes, and finally to select the most important attribute. This task presumably forced individuals to make trade-offs first within attributes (selecting the most attractive level of each attribute) and then across attributes (selecting the most important attribute). In contrast, respondents in the no-trade-off condition were asked only to evaluate the at- 
tractiveness of each level of all attributes (100-point scale: $0=$ not attractive at all; $100=$ very attractive) and the importance of each attribute (100-point scale: $0=$ not important at all; $100=$ very important). Each attribute level was ranked independently from the others, and a subject could rate all levels of an attribute as 100 points (very attractive) as well as rate all attributes as 100 points (very important). Thus, respondents in this condition were asked to evaluate all attributes, but without making explicit tradeoffs among the levels of each attribute.

Following the preference-articulation procedure, respondents were asked to choose from two sets: a small assortment (four chocolates) and a large assortment (16 chocolates). The stimuli and the rest of the experimental procedure were the same as those used in the first experiment.

\section{Results}

The data illustrating respondents' switching behavior are given in figure 2 . Respondents who were asked to articulate their attribute preferences without making explicit trade-offs were more likely to switch to the most popular alternative when choosing from the large compared to the small assortment ( $45 \%$ vs. $19 \%)$. In contrast, respondents who were asked to trade off attributes and attribute levels prior to choice displayed a trend in the opposite direction. For these respondents, choices from smaller sets were more likely to result in choosing the default alternative compared to choices from larger sets (17\% vs. $31 \%)$.

Categorical analysis of the data shows that the moderating impact of the type of articulation is significant $\left(\chi^{2}(1)=\right.$ $4.02, p<.05)$. These data are consistent with the predictions made by hypothesis 1 . For respondents in the no-ideal-point condition, the effect of product assortment was marginally significant $\left(\chi^{2}(1)=4.42, p=.06\right)$. For respondents in the ideal point condition, the effect was in the opposite direction, although nonsignificant $\left(\chi^{2}(1)=1.0, \mathrm{NS}\right)$.

More important, for choices from large assortments, the impact of the type of the preference-articulation procedure was significant $\left(17 \%\right.$ vs. $\left.45 \% ; \chi^{2}(1)=4.00, p<.05\right)$, indicating that respondents who were given the explicit task of forming an ideal point were less likely to select the default alternative compared to respondents who were required to evaluate alternatives without forming an ideal point (hypothesis 2). For choices from small assortments, the impact of the preference-articulation procedure was directionally consistent with the predictions made by hypothesis 3 , but nonsignificant $\left(31 \%\right.$ vs. $19 \%$; $\chi^{2}<1$, NS).

These data are consistent with the proposition that ideal point availability moderates the impact of assortment on strength of preferences (hypothesis 1). Respondents who articulated their attribute preferences by making explicit attribute trade-offs prior to choice displayed stronger preferences for the alternative chosen from a larger set compared to respondents who were not explicitly asked to articulate their ideal attribute combination (hypothesis 2). Finally, for choices from smaller assortments, ideal point availability
FIGURE 2

SWITCHING BEHAVIOR AS A FUNCTION OF ASSORTMENT AND IDEAL POINT AVAILABILITY (EXPERIMENT 2)

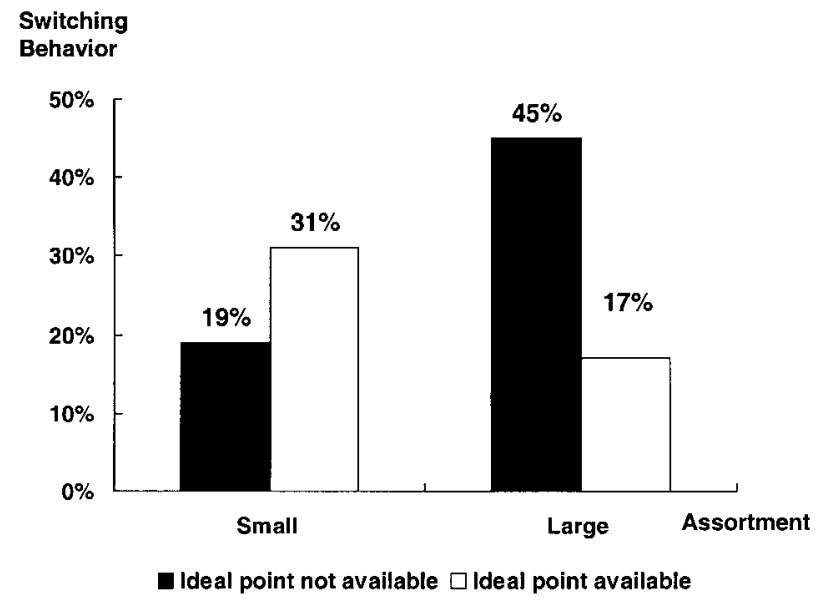

indicated a nonsignificant trend toward weakening preferences (hypothesis 3).

\section{Discussion}

Experiment 2 compared the impact of assortment on strength of preferences in two scenarios: one in which respondents were explicitly asked to articulate their ideal attribute combination and one in which they had to articulate their attribute preferences without explicitly articulating an ideal point. In this context, it was assumed that respondents who were not explicitly asked to articulate their ideal point did not have a readily available ideal attribute combination. It is possible, however, that some of the respondents in that condition articulated their ideal points spontaneously. A closer examination of the dispersion of preferences in the no-trade-off condition shows that some respondents displayed a pattern of preferences that is indicative of a hierarchical attribute structure whereby (1) one particular attribute level was rated significantly higher than others, and (2) one attribute was also considered to be more important than the others. Although not necessarily equivalent to articulating an ideal attribute combination, this pattern of dispersion of attribute preferences can be viewed as indicative of an available ideal point and used to examine the impact of assortment on choice.

Conceptually, the impact of spontaneously articulated ideal points on assortment and choice could have been analyzed by examining the readily available data from experiment 2 by comparing the dispersion of preferences for respondents in the no-trade-off condition. Given the relatively small number of observations per cell in that experiment, however, such analysis is not practically feasible. Hence, experiment 3 was designed to test how spontaneously articulated ideal attribute preferences moderate the impact of assortment on choice. 


\section{EXPERIMENT 3}

The goal of experiment 3 was to provide further support for the experimental hypotheses in a context where the ideal attribute combination is formed spontaneously rather than being experimentally induced. A secondary goal was to extend the validity of the experimental results by testing the observed effects across different product categories.

\section{Method}

One hundred sixty-seven Northwestern University students were assigned to the conditions of a 2 (ideal point availability) $\times 2$ (assortment) between-subjects factorial design. The overall experimental procedure was similar to the one used in the first two experiments. Respondents were first asked to articulate their preferences, then make a choice from either a large or a small set, and finally were given an option to reconsider their choice.

Stimuli. Unlike prior studies, in this experiment four product categories were used as stimuli: Caribbean vacation, sofa, refrigerator, and computer carrying case. Choice options were described on four attributes with either three or four levels each. Caribbean vacation was described based on location (Bahamas, Bermuda, Antigua, Jamaica), architectural style of the resort (Mediterranean, Spanish, modern), leisure activities (golf, water sports, hiking, tennis), and pool shape (oval, rectangular, kidney-shaped). The sofa was described in terms of color (black, brown, gray, white), upholstery (leather, cotton blend, velvet, suede), cushion type (firm, medium, soft), and back height (high, medium, low). The refrigerator was described in terms of the brand name (Kitchen Aid, Kenmore, Frigidaire, Maytag), color (black, almond, stainless steel, white), freezer style (top, bottom, side-by-side), and special features (in-door water dispenser, in-door ice dispenser, in-door access panel). Finally, the computer carrying case was described based on brand name (Kenneth Cole, Targus, Belkin, Kensington), bag style (saddlebag, backpack, briefcase), material (leather, nylon, fabric), and color (black, blue, red, platinum). For each product category, two assortments were constructed: a smaller set (six alternatives) and a larger set ( 24 alternatives). All choice sets were created by a random assignment of the attribute levels to choice alternatives.

Ideal Point Availability. All respondents were given a rating preference-articulation task similar to the one used in experiment 2 (no-ideal-point condition): they were asked to evaluate the attractiveness of each level of the attributes of the choice options using a 100-point rating scale. Similar to experiment 2, each attribute level was ranked independently from the others. Based on the pattern of their attribute preferences, subjects' responses were categorized into one of the two ideal point conditions. Responses that singled out one particular attribute level to be significantly more attractive than the others were considered to have an available ideal point, whereas individuals who indicated two or more attribute levels to be close in their attractiveness were considered to not have an available ideal point. The ideal point availability evaluation procedure is described in more detail in the Results section.

Experimental Procedure. For each product category, respondents were asked to articulate their preferences using an attribute-rating task and were then presented with either a small or a large assortment. Upon making a choice, respondents were asked to rate the perceived assortment (large vs. small) and then were given the option to reconsider their choice. The key dependent variable-strength of preferences-was operationalized in terms of respondents' probability of substituting their choice with an option recommended by the sales associate (e.g., "Imagine that you have to choose between the sofa you just selected and a sofa that has been recommended by the sales associate. Which one would you buy?").

\section{Results}

Manipulation Check. For the purposes of a manipulation check, respondents' perceptions of the assortment size were measured (10-point scale: $1=$ small assortment, 10 $=$ large assortment). The data show that respondents perceived the larger set to offer significantly greater assortment compared to the smaller set $(M=7.06$ vs. $M=4.73$, $F(1,166)=39.94, p<.001)$. These data indicate that respondents indeed perceived the choice sets to vary in terms of assortment, and that the assortments used as stimuli were not perceived to be extreme in either direction.

Ideal Point Availability. Unlike prior studies, in this experiment respondents were not explicitly asked to articulate their ideal point, and the ideal point availability was inferred by comparing individuals' attribute-level preferences captured by the preference-articulation task. Thus, each respondent's within-attribute evaluations were compared to determine the strength of attribute-level preferences.

Because the availability of an articulated ideal point implies that its values aggregated across all relevant attributes are superior to any other attribute combination, it was operationalized based on the proximity of the highest valued attribute level to the next highest valued one. Thus, for each attribute a differentiation score was calculated based on the difference between the highest rating given to an attribute level and the next highest one. To illustrate, the differentiation score for the response pattern 80-100-70-90 was 10, whereas the response pattern 100-60-100-100 was quantified with a differentiation coefficient of zero. Thus, the differentiation score for each attribute potentially varied between zero and 100. Next, a cumulative articulation score was calculated by aggregating the attribute-specific scores.

Based on their cumulative articulation scores, respondents were assigned to one of the two ideal point conditions using a median split. Eighty-one respondents were classified in the ideal point condition (46 in the large-assortment condition and 40 in the small-assortment condition), and the remaining 86 were classified in the no-ideal-point condition 
(38 in the large-assortment condition and 43 in the smallassortment condition). Note that this classification is for comparison purposes only, and, conceptually, the underlying distribution of ideal point articulation scores is continuous in nature.

Switching Behavior. The data show a pattern similar to the one reported in figure 2. The impact of ideal point availability on switching behavior varied as a function of the assortment, as indicated by the significant interaction effect $\left(\chi^{2}(1)=5.16, p<.05\right)$ - a finding consistent with hypothesis 1 . This effect is consistent across the product categories, as shown by the nonsignificant three-way interaction of product category, assortment, and ideal point availability $\left(\chi^{2}<1\right.$, NS).

In choices from large assortments, respondents with a high ideal point availability score (IPAS) were less likely to switch than those with a low IPAS (11\% vs. $35 \%$; $\left.\chi^{2}(1)=6.09, p<.05\right)$. This finding is consistent with hypothesis 2 . In contrast, for choices made from smaller assortments, the effect was in the opposite direction, although nonsignificant: high-IPAS respondents were less likely to switch than were the low-IPAS respondents (21\% vs. $15 \%$; $\chi^{2}<1$, NS). This finding is directionally consistent with hypothesis 3 .

Further analysis shows that respondents with a low IPAS were more likely to switch after making a choice from a larger rather than a smaller assortment (35\% vs. $15 \%$; $\left.\chi^{2}(1)=4.19, p<.05\right)$. The corresponding data pattern of responses for individuals with a high IPAS was reversed and nonsignificant $\left(11 \%\right.$ vs. $21 \% ; \chi^{2}(1)=1.57$, NS). These data are consistent with the findings reported by prior experiments, lending further support to the experimental hypotheses.

Finally, to validate the robustness of the above findings, two alternative methodologies for calculating the IPAS were tested. The first methodology applied an adjustment for the variance of individuals' preferences for all attribute levels (recall that the IPAS model presented above focused only on the difference between the highest and the next highest level). The second methodology used interval scaling, whereby the difference between the two top attribute values was classified into four categories based on the magnitude of their differences; these interval scores were then aggregated to yield the overall IPAS scores. Both methods yielded data that converged with the above findings in terms of the direction and the significance of the effects.

\section{Discussion}

The data reported in this experiment support the proposition that ideal point availability moderates the impact of assortment on choice. When choosing from a large assortment, consumers whose attribute preferences indicated a well-defined ideal point were more confident in their decisions and less likely to switch than consumers whose preferences did not imply a single ideal point. These findings are consistent with the proposition that a readily formed ideal attribute combination provides consumers with a benchmark for evaluating the alternatives in the choice set, and, as a result, for these consumers choices from large assortments are less likely to be associated with weaker preferences.

The data also show that consumers without a readily available ideal attribute combination are more likely to be susceptible to a cognitive overload when evaluating large assortments even if they have readily articulated nonhierarchical attribute evaluations. This finding is particularly interesting because intuitively one might expect that articulated preferences should always help the decision process. One explanation for the observed data pattern is offered by cognitivedissonance research, which implies that in some cases articulated attribute evaluations may actually complicate consumer choice (Festinger 1964; Janis and Mann 1977). To illustrate, consider a consumer who views two levels of the same attribute as equally attractive. When asked to make a choice, this consumer might find it difficult to trade off these attribute levels because the preference-articulation task has made salient the fact that these attributes are equally attractive. In this context, consumers who rated two or more attributes as very attractive have essentially made a commitment to both attribute levels, which in turn makes the cognitive dissonance associated with rejecting either of them more pronounced. This cognitive dissonance, in turn, is likely to lower consumers' decision confidence and increase the probability of switching.

All of the experiments presented so far examine how ideal point availability moderates the impact of assortment on choice in a context where consumers were given the attribute-level information prior to choice. An argument that could potentially limit the validity of the experimental results is that the prechoice availability of the attribute information could have structured individuals' decision processes or could have affected their level of involvement in a way that enhances the impact of ideal point availability on assortment and choice. Indeed, familiarity with the attribute dimensionality of the choice task has been shown to facilitate further learning and task performance (West, Brown, and Hoch 1996). Respondents who were given a consumption vocabulary (i.e., were told about the relevant attributes prior to evaluating alternatives) performed better than subjects who had not been initially exposed to attribute information. It has also been shown that consumers who are aware of the dimensionality of the choice set might perceive the set to be less complex because they understand the underlying structure of the set (Alba and Hutchinson 1987; Bettman and Park 1980). As a result, the mere exposure to choice attributes could have simplified the consumer decision task, potentially moderating the impact of product assortment on consumer choice (Bettman 1975). It is important, therefore, to test whether predicted ideal point availability effects hold in a scenario where respondents are not given specific attribute-level information prior to choice. 


\section{EXPERIMENT 4}

Experiment 4 tests the hypothesis that ideal point availability moderates the impact of assortment on choice by using a manipulation that does not impose a predetermined attribute structure on the consumer decisions. For that purpose, respondents were asked to articulate their ideal attribute combination using subjectively generated attribute levels rather than being given the actual attribute levels describing choice alternatives.

\section{Method}

One hundred sixty-eight Northwestern University students were presented with a 2 (ideal point availability) $\times$ 2 (assortment) mixed factorial design. The stimuli were identical to the ones used in experiment 3 (Caribbean vacation, sofa, refrigerator, and computer carrying case). Thus, in each product category there were two assortments: a smaller set (six alternatives) and a larger set (24 alternatives).

Ideal Point Availability. Respondents in the articulation condition were asked to think about their ideal product, but unlike in prior experiments, they were not provided with specific attribute levels. Thus, respondents were given information only about the attribute dimensions describing each of the alternatives, and were not forced to articulate their preferences along those dimensions. To illustrate, in the sofa category, respondents were given the following instruction: "Imagine that you are buying a sofa. Think about what your ideal sofa looks like (e.g., in terms of color, upholstery, back height, cushion softness). Please, describe your ideal sofa in the space below." In contrast, respondents in the no-articulation condition were asked to imagine that they were buying a sofa and were informed that they would be shown a selection of sofas that vary on several attributes (e.g., color, upholstery, back height, and cushion softness) but were not asked to generate their ideal attribute combination.

Experimental Procedure. Respondents were first given the ideal point availability task, asked to make a choice from either a large or a small set, and then given an option to switch. Next they were presented with a different choice set in the same product category that varied in terms of assortment. Thus, respondents who were initially asked to make a choice from a larger set now had to choose from a smaller set and vice versa. This procedure was repeated across the ideal point availability conditions: individuals who were initially not asked to articulate their ideal point were now in the ideal point condition. To avoid potential carry-over attribute learning effects, the second set of choices was made from a different product category. Thus, each individual made four choices, two in each product category. The order of assortments and ideal point availability conditions was counterbalanced across the individuals.

Dependent Variables. Similar to experiment 3, the dependent variable — strength of preferences-was operation- alized in terms of respondents' probability of substituting their choice with an option recommended by the sales associate. An additional measure of the strength of preferences was respondents' confidence in their decisions measured on a 10-point scale (e.g., "How would you rate your confidence that you will actually like the sofa you selected?" $1=$ not confident at all, $10=$ very confident). Respondents' evaluations of perceived decision difficulty were measured as well (e.g., "How would you rate the difficulty of this decision for you?" 10 -point scale: $1=$ not difficult at all, 10 $=$ very difficult).

\section{Results}

The manipulation check confirmed that the assortment manipulation was successful; respondents perceived the larger set to offer significantly greater assortment compared to the smaller set $(M=7.75$ vs. $M=4.32, F(1,670)=$ $169.43, p<.001)$. The analysis of respondents' switching behavior that appears below is followed by an analysis of their confidence in the decisions made and the perceived decision difficulty.

Switching Behavior. Each of the 168 respondents made four choices, yielding 671 observations (one missing data point). These observations were equally distributed across the experimental conditions, with 168 (167) data points per condition. The data pattern was similar to the pattern presented in figure 1 . When making a choice from a large assortment, respondents who were asked to articulate their ideal attribute combination were less likely to switch than those in the no-ideal-point condition (14\% vs. $24 \%$; $\left.\chi^{2}(1)=4.59, p<.05\right)$. The corresponding pattern of responses for choices from a small assortment was reversed (19\% vs. $17 \%$; $\chi^{2}<1$, NS). These data are consistent with the predictions made by hypothesis 2 . Analysis of these data shows that the response patterns across the experimental conditions were different, as indicated by the significance of the interaction effect $\left(\chi^{2}(1)=3.97, p<.05\right)$. This finding is consistent with hypothesis 1 . This effect was consistent across the product categories, and although there was a significant main product-category effect $\left(\chi^{2}(3)=16.03\right.$, $p<.001)$, the relevant three-way interaction of product category, assortment, and articulation was nonsignificant $\left(\chi^{2}<1\right.$, NS).

Further analysis of the data revealed marginally significant sequence effects, indicating differences in the effects of ideal point availability and assortment on preferences across respondents' first and second choice from a given product category $\left(\chi^{2}(1)=2.90, p<.10\right)$. Thus, unlike the first choice, where the interaction between ideal point articulation and assortment was significant $\left(\chi^{2}(1)=5.1\right.$, $p<.05)$, in the second choice this interaction was nonsignificant $\left(\chi^{2}<1\right.$, NS), indicating that in the latter case ideal point articulation did not moderate the impact of assortment on choice. Specifically, respondents' first choice produced a pattern of responses similar to the one reported in prior experiments: for choices made from large assortments, re- 
spondents with an articulated ideal point were less likely to switch compared to those in the no-ideal-point condition $\left(17 \%\right.$ vs. $\left.33 \% ; \chi^{2}(1)=3.72, p=.05\right)$, whereas for choices from small assortments the effect was reversed and nonsignificant $\left(10 \%\right.$ vs. $\left.17 \% ; \chi^{2}(1)=1.83, p<.20\right)$. This pattern of results is consistent with the predictions made by hypotheses 2 and 3 . In contrast, when respondents made their second selection from the same product category, these effects were less pronounced and nonsignificant: when choosing from large assortments, respondents with an articulated ideal point were less likely to switch $\left(11 \%\right.$ vs. $14 \% ; \chi^{2}<$ $1, \mathrm{NS}$ ), and a similar result was obtained for choices from small assortments $\left(21 \%\right.$ vs. $25 \% ; \chi^{2}<1$, NS). These unpredicted yet significant sequence effects are addressed in more detail in the Discussion section.

Decision Confidence. Each of the 168 respondents made four choices, yielding 672 observations (no missing observations). The data presented in figure $3 A$ show that when choosing from a large assortment, respondents with an available ideal point were more confident in their decisions compared to respondents who did not articulate their ideal attribute combination $(M=7.58, \mathrm{SD}=1.77$ vs. $M=7.21, \mathrm{SD}=2.06)$. In contrast, when choosing from a small assortment, the effect was reversed, whereby respondents with an available ideal point were less confident in their choices $(M=7.02, \quad \mathrm{SD}=1.90$ vs. $M=7.54$, $\mathrm{SD}=1.83)$. Analysis of these data (ANOVA) shows that these effects are significant $(F(1,480)=3.84, p=.05$ and $F(1,480)=7.11, p<.01$, respectively). These data support hypotheses 2 and 3 . The data further show that ideal point availability moderates the impact of assortment on confidence, as indicated by the significant interaction $(F(1,480)$ $=10.69, p<.005)$-a result consistent with hypothesis 1 .

Furthermore, there was a significant sequence effect, indicating that individuals' responses varied depending on whether they were making their first or second choice from a given product category $(F(3,480)=4.93, p<.005)$. Thus, the (ideal point availability) $\times$ (assortment) interaction effect reported above was more pronounced in the context of respondents' first than second choice $(F(1,480)=11.86, p<.001$ vs. $F(1,480)=2.79, p<$ .10). Specifically, individuals who were not asked to articulate their preferences were less confident when choosing from a large relative to a small assortment when they made their first selection $(M=7.64, \mathrm{SD}=1.76$ vs. $M=6.92$, $\mathrm{SD}=2.32, F(1,480)=7.51, p<.01)$ than in their second selection $(M=7.45, \mathrm{SD}=1.90$ vs. $M=7.5, \mathrm{SD}=$ $1.72, F<1$, NS), whereas for respondents with an articulated ideal point the sequence effect was less pronounced $(F<1, \mathrm{NS})$. Thus, the predicted ideal point availability effects were more prominent in respondents' first choice decisions and less pronounced in the subsequent choice from the same category - a result further conceptualized in the Discussion section.

Decision Difficulty. The data presented in figure $3 B$ offer additional evidence in support of the experimental hy-
FIGURE 3

\section{DECISION CONFIDENCE AND DECISION DIFFICULTY AS A FUNCTION OF ASSORTMENT AND IDEAL POINT AVAILABILITY (EXPERIMENT 4)}

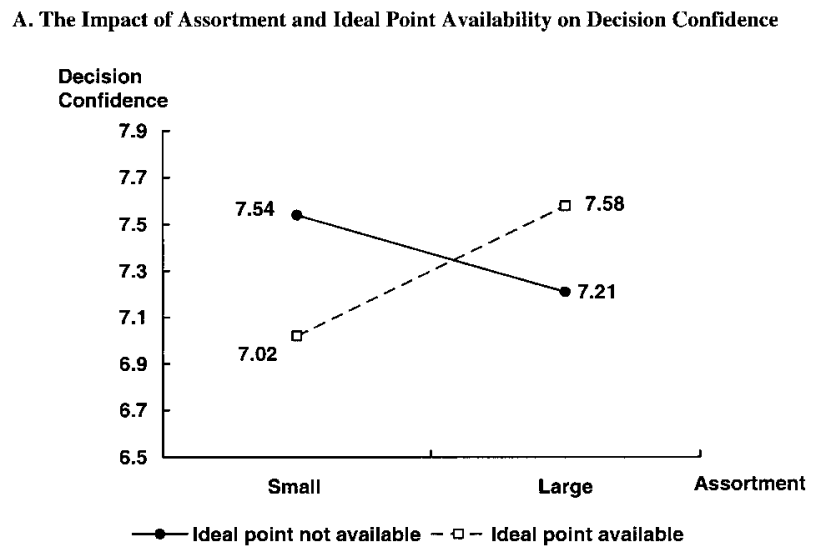

B. The Impact of Assortment and Ideal Point Availability on Decision Difficulty

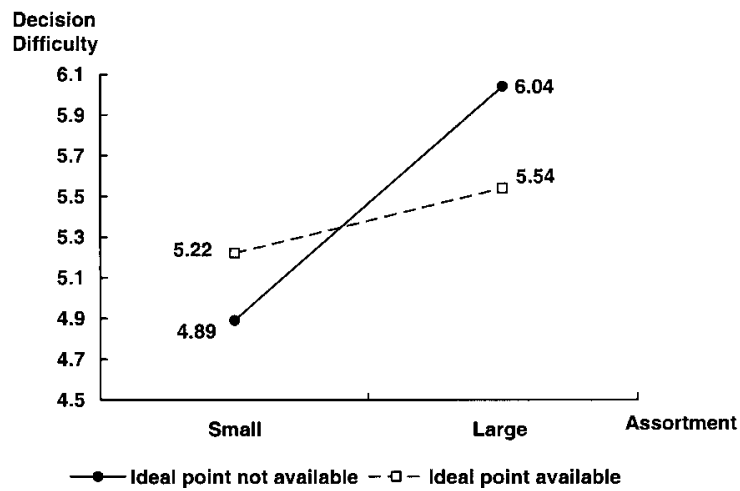

potheses. The data show that choices from large assortments were perceived to be more difficult by respondents who were not asked to articulate their ideal point compared to respondents who articulated their ideal attribute combination $(M=6.04, \quad \mathrm{SD}=2.45 \quad$ vs. $\quad M=5.54, \quad \mathrm{SD}=2.54$; $F(1,480)=4.95, p<.05)$, whereas for choices from small assortments the effect was reversed $(M=4.89, \mathrm{SD}=$ 2.23 vs. $M=5.22, \mathrm{SD}=2.28 ; F(1,480)=1.50, \mathrm{NS})$. The main effect of assortment on decision difficulty was significant $(F(1,480)=13.36, p<.001)$, indicating that, overall, choosing from a large assortment was perceived to be relatively more difficult than choosing from a small assortment. More important, there was a significant interaction $(F(1,480)=5.94, p<.01)$, indicating that the impact of assortment on the decision difficulty was moderated by the availability of an ideal point. Finally, unlike with decision confidence, there were no order effects; the decision-difficulty statistics for sequential choices made from the same product category were nearly identical. 


\section{Discussion}

The data show an interesting pattern whereby the hypothesized effects were more pronounced for the first choices made from a given product category and less pronounced for subsequent choices. One rationale for these data is that when making a choice from smaller assortments individuals implicitly formed an ideal attribute combination, which they later used to evaluate options in the larger assortment presented to them. Thus, the implicit ideal point articulation in the first choice could have resulted in implicit learning of ideal points that were used to guide subsequent decisions.

A further observation is that, whereas the switching and decision confidence data displayed a learning pattern across sequential choices, the decision-difficulty data did not reflect such effects. One possible explanation for these data is that making a choice from a smaller set did not necessarily familiarize individuals with the structure of the larger set over and above the information readily available to all respondents in the prechoice articulation task. Similarly, respondents who initially made a choice from a larger set perceived the choice from a smaller set to be perceptually easier, yet more difficult in terms of locating an alternative that best matched the implicitly articulated ideal created by the first selection. Thus, making an initial choice did not necessarily change respondents' perceptions of the difficulty of the subsequent choice decision.

In general, the data from this experiment lend further support to the theory and experimental hypotheses advanced in this research. The decision confidence and difficulty data offer insights into decision processes underlying consumer choices and offer converging evidence in support of the theory. Overall, the data show that articulating an ideal point moderates the impact of assortment on choice even when consumers subjectively generate the attribute-rating metrics to form their ideal attribute combination. The use of this alternative preference-elicitation procedure offers further evidence that the observed preference-articulation effects cannot be accounted for by respondents' familiarity with the particular attribute structure of the alternatives in the choice set and the perceived complexity of the choice task associated with it. Considered together with the other studies reported in this article, this experiment confirms prior findings and validates the experimental hypotheses in a broader context.

\section{GENERAL DISCUSSION}

Research presented in this article demonstrates that, contrary to the common wisdom that more choice is always better, selections made from large assortments can lead to weaker preferences. Building on the extant literature, this research identifies ideal point availability as a key factor that determines when large assortments will strengthen consumer preferences and when large assortments will weaken preferences (hypothesis 1). It is theorized that consumers without an ideal point face the relatively complex task of simultaneously forming their ideal attribute combination and searching for the option that best matches their ideal point. It is therefore proposed that, for choices made from large assortments, ideal point availability can simplify choice, leading to a stronger preference for the selected alterative (hypothesis 2). Ideal point availability can also have the opposite impact on preferences if a close match is not available - a scenario more likely to occur in the context of a small rather than a large assortment. It is therefore argued that, for choices made from smaller assortments, ideal point availability can have the opposite effect, leading to weaker preferences for the selected alternative (hypothesis 3).

The data from four experiments offer converging evidence in support of the experimental hypotheses. The moderating effect of the ideal point availability hypothesis (hypothesis 1) is supported in all four experiments. The proposition that ideal point availability tends to strengthen preferences in choices from large assortments (hypothesis 2) received consistent support across experiments as well. The proposition that ideal point availability can have an adverse impact on strength of preferences in choices from small assortments (hypothesis 3) received directional support in all experiments. When aggregated across experiments, the evidence in support of hypothesis 3 is also significant (Edgington 1972; Rosenthal 1978). Considered together, these experiments lend support for all experimental propositions.

In addition to the formally stated experimental hypotheses, this research documents the role of availability of articulated attribute trade-offs as a key factor moderating the impact of assortment on choice. Thus, respondents who were explicitly asked to articulate attribute trade-offs were more confident in their decisions and less likely to switch compared to consumers who articulated their preferences without making explicit attribute trade-offs. This finding further contributes to the decision literature demonstrating that learning trade-offs is a key component of articulating the ideal attribute combination (Carpenter and Nakamoto 1989; Luce, Payne, and Bettman 1999; Simonson and Tversky 1992).

The findings reported in this research raise the question of how ideal point availability affects a consumer's selection of choice heuristics in the context of different assortments. Prior research has shown that information search is less complete, more selective, and more attribute based as the number of options increases (Payne 1976; Payne and Braunstein 1978; Timmermans 1993). Building on the notion that people evaluate choice alternatives relative to a reference point (Tversky and Kahneman 1991), it can be argued that consumers with articulated preferences are more likely (relative to those without articulated preferences) to use their ideal attribute combination as a reference point to evaluate choice alternatives. Ideal point availability can also lead to confirmatory processes that are characterized by reliance on positive-test strategies and selective processing of the available information (Chernev 2001; Klayman and Ha 1987; Russo, Meloy, and Medvec 1998). Building on this research, one can argue that choices made from large assortments are 
likely to be associated with more selective, alternativebased, and confirmatory processing for individuals with an articulated ideal attribute combination and more comprehensive, attribute-based, and comparative processing for those without articulated preferences (Chernev 2003). Investigating the role of ideal point availability on a consumer's decision strategy is a venue for further research that will likely provide valuable insights into the role of assortment in preference formation.

[David Glen Mick served as editor and Michael D.

Johnson served as associate editor for this article.]

\section{REFERENCES}

Alba, Joseph W. and J. Wesley Hutchinson (1987), "Dimensions of Consumer Expertise," Journal of Consumer Research, 13 (March), 411-455.

Bettman, James R. (1975), "Issues in Designing Consumer Information Environments," Journal of Consumer Research, 2 (December), 169-177.

- (1979), An Information Processing Theory of Consumer Choice, Reading, MA: Addison-Wesley.

Bettman, James R., Mary Frances Luce, and John W. Payne (1998), "Constructive Consumer Choice Processes," Journal of Consumer Research, 25 (December), 187-217.

Bettman, James R. and C. Whan Park (1980), "Effects of Prior Knowledge and Experience and Phase of the Choice Process on Consumer Decision Processes: A Protocol Analysis," Journal of Consumer Research, 7 (December), 234-248.

Bettman, James R. and Michel A. Zins (1979), "Information Format and Choice Task Effects in Decision Making," Journal of Consumer Research, 6 (September), 141-153.

Biehal, Gabriel and Dipankar Chakravarti (1982), "InformationPresentation Format and Learning Goals as Determinants of Consumers' Memory Retrieval and Choice Processes," Journal of Consumer Research, 8 (March), 431-441.

Brehm, Jack W. (1972), Responses to the Loss of Freedom: A Theory of Psychological Reactance, Morristown, NJ: General Learning Press.

Broniarczyk, Susan M., Wayne D. Hoyer, and Leigh McAlister (1998), "Consumers' Perceptions of the Assortment Offered in a Grocery Category: The Impact of Item Reduction," Journal of Marketing Research, 35 (September), 166-176.

Calder, Bobby J. (1978), "Cognitive Response, Imagery and Scripts: What Is the Cognitive Basis of Attitude?" in Advances in Consumer Research, Vol. 5, ed. H. Keith Hunt, Ann Arbor, MI: Association for Consumer Research.

Carpenter, Gregory S. and Kent Nakamoto (1989), "Consumer Preference Formation and Pioneering Advantage," Journal of Marketing Research, 26 (August), 285-298.

Chernev, Alexander (2001), "The Impact of Common Features on Consumer Preferences: A Case of Confirmatory Reasoning," Journal of Consumer Research, 27 (March), 475-488.

- (2003), "Product Assortment and Individual Decision Processes," Journal of Personality and Social Psychology, 85 (July), 151-162.

Dhar, Ravi (1997), "Consumer Preference for a No-Choice Option," Journal of Consumer Research, 24 (September), 215-231.

Edgington, Eugene S. (1972), “An Additive Method for Combining
Probability Values from Independent Experiments," Journal of Psychology, 80 (March), 351-363.

Festinger, Leon (1964), Conflict, Decision, and Dissonance, Stanford, CA: Stanford University Press.

Garbarino, Ellen C. and Julie A. Edell (1997), "Cognitive Effort, Affect, and Choice," Journal of Consumer Research, 24 (September), 147-158.

Green, Paul E., Abba M. Krieger, Manoj K. Agarwal, and Richard M. Johnson (1991), "Adaptive Conjoint Analysis: Some Caveats and Suggestions," Journal of Marketing Research, 28 (May), 215-226.

Green, Paul E. and V. Srinivasan (1990), "Conjoint Analysis in Marketing: New Developments with Implications for Research and Practice," Journal of Marketing, 54 (4), 3-19.

Greenleaf, Eric A. and Donald R. Lehmann (1995), "Reasons for Substantial Delay in Consumer Decision Making," Journal of Consumer Research, 22 (September), 186-199.

Hoch, Stephen J., Eric T. Bradlow, and Brian Wansink (1999), "The Variety of an Assortment," Marketing Science, 18 (4), 527-546.

Holbrook, Morris B. and William L. Moore (1981), "Feature Interactions in Consumer Judgments of Verbal versus Pictorial Presentations," Journal of Consumer Research, 8 (June), 103-113.

Huber, Joel, Dick R. Wittink, John A. Fiedler, and Richard Miller (1993), "The Effectiveness of Alternative Preference Elicitation Procedures in Predicting Choice," Journal of Marketing Research, 30 (February), 105-214.

Huffman, Cynthia and Barbara E. Kahn (1998), "Variety for Sale: Mass Customization or Mass Confusion?" Journal of Retailing, 74 (4), 491-513.

Iyengar, Sheena S. and Mark R. Lepper (2000), "When Choice Is Demotivating: Can One Desire Too Much of a Good Thing?" Journal of Personality and Social Psychology, 79 (6), 995-1006.

Jacoby, Jacob, Donald E. Speller, and Carol A. Kohn (1974), "Brand Choice Behavior as a Function of Information Load," Journal of Marketing Research, 11 (February), 63-69.

Janis, Irving L. and Leon Mann (1977), Decision Making: A Psychological Analysis of Conflict, Choice, and Commitment, New York: Free Press.

Kahn, Barbara E. and Donald R. Lehmann (1991), "Modeling Choice among Assortments," Journal of Retailing, 67 (3), 274-299.

Kahn, Barbara, William L. Moore, and Rashi Glazer (1987), "Experiments in Constrained Choice," Journal of Consumer Research, 14 (June), 96-113.

Karni, Edi and Aba Schwartz (1977), "Search Theory: The Case of Search with Uncertain Recall," Journal of Economic Theory, 16 (1), 38-52.

Klayman, Joshua and Young Ha (1987), "Confirmation, Disconfirmation, and Information in Hypothesis Testing," Psychological Review, 94 (2), 211-228.

Kottemann, Jeffrey E. and Fred D. Davis (1991), "Decisional Conflict and User Acceptance of Multicriteria Decision-Making Aids," Decision Sciences, 22 (4), 918-926.

Kreps, David M. (1979), "A Representation Theorem for 'Preference for Flexibility'," Econometrica, 47 (3), 565-577.

Lancaster, Kelvin (1990), "The Economics of Product Variety: A Survey," Marketing Science, 9 (3), 189-206.

Luce, Mary Frances, John W. Payne, and James R. Bettman (1999), "Emotional Trade-Off Difficulty and Choice," Journal of Marketing Research, 36 (May), 143-159. 
Malhotra, Naresh K. (1982), "Information Load and Consumer Decision Making," Journal of Consumer Research, 8 (March), 419-430.

March, James G. (1978), "Bounded Rationality, Ambiguity, and the Engineering of Choice," Bell Journal of Economics, 9 (2), 587-608.

McAlister, Leigh (1982), "A Dynamic Attribute Satiation Model of Variety-Seeking Behavior," Journal of Consumer Research, 9 (September), 141-151.

Miller, George A. (1956), "The Magical Number Seven, Plus or Minus Two: Some Limits on Our Capacity for Processing Information," Psychological Review, 63, 81-97.

Payne, John W. (1976), "Task Complexity and Contingent Processing in Decision Making: An Information Search and Protocol Analysis," Organizational Behavior and Human Decision Processes, 16 (2), 366-387.

Payne, John W., James R. Bettman, and Eric J. Johnson (1993), The Adaptive Decision Maker, New York: Cambridge University Press.

Payne, John W. and Myron L. Braunstein (1978), "Risky Choice: An Examination of Information Acquisition Behavior," Memory and Cognition, 6 (5), 554-561.

Pessemier, Edgar A. (1978), "Stochastic Properties of Changing Preferences," American Economic Review, 68 (2), 380-385.

Ratner, Rebecca K., Barbara E. Kahn, and Daniel Kahneman (1999), "Choosing Less-Preferred Experiences for the Sake of Variety," Journal of Consumer Research, 26 (June), 1-15.

Reibstein, David J., Stuart A. Youngblood, and Howard L. Fromkin (1975), "Number of Choices and Perceived Decision Freedom as a Determinant of Satisfaction and Consumer Behavior," Journal of Applied Psychology, 60 (4), 434-437.

Rosenthal, Robert (1978), "Combining Results of Independent Studies," Psychological Bulletin, 85 (January), 185-193.

Russo, J. Edward, Margaret Meloy, and Victoria Husted Medvec (1998), "Predecisional Distortion of Product Information," Journal of Marketing Research, 35 (November), 438-452.
Scammon, Debra L. (1977), "Information Load and Consumers," Journal of Consumer Research, 4 (March), 148-155.

Shugan, Steven M. (1980), "The Cost of Thinking," Journal of Consumer Research, 7 (September), 99-111.

Simonson, Itamar (1990), "The Effect of Purchase Quantity and Timing on Variety-Seeking Behavior," Journal of Marketing Research, 27 (May), 150-162.

- (1999), "The Effect of Product Assortment on Buyer Preferences," Journal of Retailing, 75 (3), 347-370.

Simonson, Itamar and Amos Tversky (1992), "Choice in Context: Tradeoff Contrast and Extremeness Aversion," Journal of Marketing Research, 29 (August), 281-295.

Timmermans, Danielle (1993), "The Impact of Task Complexity on Information Use in Multi-Attribute Decision Making," Journal of Behavioral Decision Making, 6 (2), 95-111.

Tversky, Amos (1977), "Features of Similarity," Psychological Review, 84 (4), 327-352.

Tversky, Amos and Daniel Kahneman (1974), "Judgment under Uncertainty: Heuristics and Biases," Science, 185 (4157), 1124-1131.

(1991), "Loss Aversion in Riskless Choice: A ReferenceDependent Model," Quarterly Journal of Economics, 106 (November), 1039-1061.

Walsh, John W. (1995), "Flexibility in Consumer Purchasing for Uncertain Future Tastes," Marketing Science, 14 (2), 148-165.

Wansink, Brian, Robert J. Kent, and Stephen J. Hoch (1998), “An Anchoring and Adjustment Model of Purchase Quantity Decisions," Journal of Marketing Research, 35 (February), 71-81.

West, Patricia M., Christina L. Brown, and Stephen J. Hoch (1996), "Consumption Vocabulary and Preference Formation," Journal of Consumer Research, 23 (September), 120-135.

Wright, Peter (1975), "Consumer Choice Strategies: Simplifying vs. Optimizing," Journal of Marketing Research, 12 (February), 60-67. 